\title{
NON-INVASIVE ASSESSMENT OF SHORT AND ULTRA-SHORT HEART RATE VARIABILITY DURING DIFFERENT PHYSICAL AND PHYSIOLOGICAL TESTS
}

\author{
Tuncay Alparslan', Ramiz Arabaci², and Recep Gorgulu ${ }^{2}$ \\ ${ }^{1}$ Aeromedical Research and Training Center, Eskişehir, Turkey \\ ${ }^{2}$ Bursa Uludăg University, Faculty of Sport Sciences Bursa, Turkey
}

Original scientific paper

DOI: $10.26582 / \mathrm{k} .53 .1 .15$

\begin{abstract}
:
The main aim of the present study was to determine the short- and ultra-short-term heart rate variability (HRV) during different physical and physiological tests and to compare HRV to different performance levels. The latter aim was to compare participants' short- and ultra-short-term heart rate variability before-, during-, and post-tests. Our hypothesis was that there would be a significant difference between test performance and HRV parameters, and the high performing group would have significantly higher HRV parameters than the low performing group. Fifty-three healthy men $\left(\mathrm{M}_{\text {age }}=26.9 \pm 4\right.$ years, $\left.\mathrm{M}_{\text {height }}=177.9 \pm 5.7 \mathrm{~cm} ; \mathrm{M}_{\text {weight }}=77.8 \pm 8.7 \mathrm{~kg}\right)$ were recruited in the current study. We completed the data collection procedure for each participant in four consecutive days. On day-1, anthropometric measurements were conducted and then participants performed isokinetic tests. On day-2, participants performed anaerobic tests; on day-3 equilibrium tests, and on day-4 aerobic capacity tests. The HRV records of all participants were obtained before, during and after all these tests. Based on the participants' performance, they were classified into two groups: participants in G1 had lower performance and those in G2 higher performance. A two-way repeated measures ANOVA yielded significant differences in HRV values obtained in the four different tests. There was a significant difference between fitness test performance groups in the variation of short- and ultra-short-term HRV parameters.
\end{abstract}

Key words: autonomic nervous system, heart rate variability, physical and physiological tests

\section{Introduction}

Heart rate variability (HRV) is an objective marker that helps to understand human performance as a measure of the continuous interplay between the sympathetic and parasympathetic influences on heart rate (Berntson, et al., 1997; Marek, 1996; Taralov, Terziyski, \& Kostianev, 2015). Of particular interest in performance contexts is that human performance can be indexed through the autonomic nervous system in the form of HRV (Buchheit, et al., 2010; Giles \& Draper, 2018; Mosley \& Laborde, 2015).

Studies on ultra-short-term HRV using less than 5-minute data sets have been performed according to the increased demands for various practical applications by means of ambulatory heart rate (HR) monitoring devices (Baek, et al., 2015; McNames \& Aboy, 2006; Nussinovitch, et al., 2011). There is a considerable amount of research in short-term and ultra-short-term HRV measurements that provide consistent results during recovery after exercise (Kiviniemi, Hautala, Kinnunen, \& Tulppo, 2007; Makivić, Nikić Djordjević, \& Willis, 2013; Seiler,
Haugen, \& Kuffel, 2007) aiming to determine the timing of athletes achieving maximum performance (Chalencon, et al., 2012; Manzi, et al., 2009; Plews, Laursen, Kilding, \& Buchheit, 2012). Pichon, De Bisschop, Roulaud, Denjean, and Papelier (2004) indicated that short-term HRV measurement in time and frequency domains was a useful tool for assessing healthy and trained males' HRV parameters. In addition, Weippert, Behrens, Rieger, Stoll, and Kreuzfeld. (2013) conducted a study using static and dynamic exercises while monitoring healthy male participants' HRV. In their investigation, results of the blood pressure and HRV analysis in time and frequency domain contributed to the assumptions that static exercises at low heart rate levels led to stronger vasoconstriction than dynamic exercises and the vagal cardiac efferent activity was enhanced during static exercise.

Researchers are continuing to link HRV domains to physical performance under different conditions and durations. On the other hand, the measurement of non-invasive HRV data is also available and interpretable by coaches and athletes 
in athletic performance settings (Tomes, Schram, \& Orr, 2020). Questions remain, however, do we precisely understand the effect of different physical activities on HRV changes under different durations such as short-term and ultra-short-term.

To our knowledge, this is the first study conducted on serial physical performance tests (pretest - test - post-test) while measuring the short and ultra-short term HRV parameters. Is there a difference in the short- and ultra-short monitoring of HRV between the groups whose performance is above the average and the group with belowaverage performance? Does HRV change before, during and after physical and physiological tests? Our hypotheses are that 1) there will be a significant difference in the short- and ultra-short-time HRV between the two groups with different performance level, and 2) there will be a significant difference among HRVs measured before, during and after the tests. Therefore, the current research aimed to address the differential effect of physical tests on short- and ultra-short-term HRV measures. More specifically, the main purpose of this study is to determine the short- and ultra-short HRV for monitoring of healthy men during different physical and physiological tests and to compare HRVs for different performance levels. The second purpose is to compare pre-test, test and post-test short- and ultra-short-term HRV.

\section{Methods}

\section{Participants}

Fifty-nine healthy males were recruited on a volunteer basis through advertisement posters. Six participants were excluded from the current study due to their busy schedule. The final sample comprised 53 individuals $\left(\mathrm{M}_{a g e}=26.9 \pm 4\right.$ years, $\left.\mathrm{M}_{\text {height }}=177.9 \pm 5.7 \mathrm{~cm} ; \mathrm{M}_{\text {weigt }}=77.8 \pm 8.7 \mathrm{~kg}\right)$. All participants were reported being free from illness and injury during the last six months and no medical drugs were used in the week before the experiments took place. Informed consent was obtained from all participants. The experiments were approved by the Eskişehir Osmangazi University research ethics committee (Approval Number: 2019/73).

Data collection for each participant was executed on weekdays during morning hours (i.e. from 10:00 a.m. to 12:00 a.m.). Moderate drinking water consumption was allowed to each participant during the tests. All measurements were performed in a quiet and air-conditioned (air temperature $22-24^{\circ} \mathrm{C}$, air humidity $33-45 \%$ ) experiment room. The participants were warned not to perform any physical activity the day before the test days and not to use stimulants such as food and medicine or coffee for two hours before the tests. The tests were performed in groups of five or six participants in four different phases so that HRV recordings did not interact with each other (Table 1). On the first day, anthropometric measurements and isokinetic dominant knee force measurements (Phase I) took place. On the second day, anaerobic power was measured by Wingate Anaerobic Test (WAnT) (Phase II). On the third day, the equilibrium test was performed (Phase III). Finally, on the fourth day, the Bruce protocol was applied on the treadmill using the gas analyzer to measure the aerobic capacity (Phase IV). HRV recordings were collected at rest for 30 minutes, which was then recorded as 60 seconds before the test, 30 seconds during the test and 60 seconds after the test for phases I-II-III, whereas for phase IV, it was five minutes pre-test and five minutes after-test. (Table 1).

Based on the results achieved in the performance tests, participants were classified into two groups as G1 (group with lower performance) and G2 (group with higher performance) (ACSM, 2013; Franklin, Whaley, \& Howley, 2000; Zupan, et al., 2009). HRV differences were observed between the participants with high performance and those with low performance.

\section{Measurements}

The tests selected for the research are the tests that are frequently used in determining the conditional properties using either indirect or direct measurement methods. The tests applied are laboratory tests that give objective results due to the protocols and devices used.

Anthropometric measurements: Body height of the participants was measured as recommended by the International Society for the Advancement of Kinanthropometry (ISAK) and with a $1 / 10 \mathrm{~cm}$ sensitivity (Holtein Harpenden 601, Holtain Ltd., UK). Body weight of the participants were measured

Table 1. Measuring times, i. e. duration of HRV records taken during the tests

\begin{tabular}{lcccc}
\hline & $\begin{array}{c}\text { Phase I } \\
\text { Day 1 10:00/12:00 } \\
\text { Isokinetic Strength } \\
\text { Test }\end{array}$ & $\begin{array}{c}\text { Phase II } \\
\text { Day 2 10:00/12:00 } \\
\text { Anaerobic Test } \\
\text { (WAnT) }\end{array}$ & $\begin{array}{c}\text { Phase III } \\
\text { Day 3 10:00/12:00 } \\
\text { Equilibrium Test }\end{array}$ & $\begin{array}{c}\text { Phase IV } \\
\text { Day 4 10:00/12:00 } \\
\text { Aerobic Resistance Test } \\
\text { (Bruce Protocol) }\end{array}$ \\
\hline Monitoring HRV & Ultra-short & Ultra-short & Ultra-short & Short \\
Pre-test & $60 \mathrm{~s}$ & $60 \mathrm{~s}$ & $60 \mathrm{~s}$ & 5 min \\
Test & $30 \mathrm{~s}$ & $30 \mathrm{~s}$ & $30 \mathrm{~s}$ & during a test \\
Post-test & $60 \mathrm{~s}$ & $60 \mathrm{~s}$ & $60 \mathrm{~s}$ & 5 min \\
\hline
\end{tabular}


with a scale of $1 / 10 \mathrm{~kg}$ using the scale model of the InBody brand 270 models (Biospace Co., S. Korea) body analyzer. To obtain the Body Mass Index (BMI) values of the participants InBody brand 270 models (Biospace Co., S. Korea) body analyzer was used, and measurements were performed according to the procedure specified in the device manual. The data obtained were recorded in $\%$.

Isokinetic test: The flexion-extension isokinetic force measurement at 60 degrees angular velocity on the dominant knee was measured with Humac Norm device for 30 seconds (Humac Norm, Computer Sports Medicine Inc., USA). The dynamometer was calibrated before the start of the study, according to the operating manual. The weight of the leg was calculated. Participants were seated in an upright position, with the backrest at $85^{\circ}$. The rotational axis of the knee was placed in line with the dynamometer axis of rotation, and $0^{\circ}$ was determined as $0^{\circ}$ of knee extension. The lever arm pad was secured just proximal to the medial malleolus, so that movement of the ankle was not constricted. Tests were performed in a predefined ROM of $90^{\circ}-0^{\circ}$. To minimize compensatory trunk movements during testing, participants were secured using stabilizing straps, according to the manufacturer's manual. During this test, participants were encouraged verbally to exert maximal effort. HRV was measured 60 seconds before the test, 30 seconds during the test and 60 seconds after the test.

Anaerobic test: The anaerobic power tests were performed by a Wattbike brand ergometer using WAnT (Wattbike WPM ModelB, Wattbike Ltd., UK). The reliability study for a 30-second rundown sprinkle in Wattbike has already been done (Driller, Argus, \& Shing, 2013). It records a calculation of the average power in each 5 -second interval of a 30 -second test and provides a peak power value based on the highest 5-second average as well as a rate of deterioration. Participants completed the same warm-up protocol as had been performed during the orientation session and then immediately began the WAnT, a 30s maximal anaerobic exercise test on a cycle ergometer against $7.5 \%$ of their body mass (Bar-Or, 1987). During this test, participants were encouraged verbally to exert maximal effort. Mean power, peak power, and relative power were measured by the WAnT. HRV was recorded one minute before the test, 30 seconds during the test and one minute after the test.

Equilibrium test: 30 seconds static equilibrium was measured by Sigma (Sigma Platforma Balansowa, AC International East SP Z O.O., Poland). The subject steps up on the platform at the level of two feet then watch the screen for 30 seconds before the beginning of the test. The platform is equipped with an independent system for the assessment of the swing angle using a specialized sensor that detects any change in the position of the platform. It processes these changes to the appropriate output signal and transmits data wirelessly in real time to a computer with the adequate software. The area covered by the test as a result of the test was recorded on the data form. HRV was recorded 60 seconds before the test, 30 seconds during the test and 60 seconds after the test.

Aerobic test: The treadmill test with the gas analyzer was used, which is accepted as the gold standard for the determination of aerobic endurance and maximal oxygen consumption. The Bruce protocol (h/p/cosmos quasar med 170-190/65, h/p/ cosmos \& medical GMBH, Germany) was used. The staged protocol began at $1.7 \mathrm{mph}$ at the $10 \%$ grade with work rate increased (speed and grade) every three minutes until maximal oxygen consumption $\left(\mathrm{VO}_{2} \mathrm{max}\right)$ was reached. Expired gas fractions (oxygen and carbon dioxide) were collected at the mouth and analyzed with a metabolic cart (Cosmed Quark CPED metabolic cart, Roma, IT). Measurements were processed in Omnia-Standalone software for Microsoft Windows version 1.4. The criteria for $\mathrm{VO}_{2}$ max were predetermined as two out of the following: if there was a plateau in oxygen consumption despite an increased work $\left( \pm 2 \mathrm{ml} . \mathrm{kg}^{-1} \cdot \mathrm{min}^{-1}\right)$; the respiratory exchange ratio of $>1.10$; and a heart rate within ten beats of the agepredicted maximum (220-age). The Bruce protocol was applied after a 10-minute warm-up period.

Heart rate variability measurement: To obtain resting HRV measurements, HRV recordings were taken from the volunteers while sitting before the tests. HRV was measured by Polar V800 GPS Sports Watch with Heart Rate Monitor and Polar H7 band (Polar Electro, Kempele, Finland). The Polar V800 is valid to detect RR intervals with an error of $0.09 \%$ and an intra-class correlation coefficient of $>0.99$ (Giles, Draper, \& Neil, 2016). The heart rate data were stored in a personal computer using Polar FlowSync Software (version 3.0.0.1337). HRV analyses were performed using Kubios HRV standard heart rate variability analysis software for Mac (Biosignal Analysis and Medical Imaging Group, Department of Physics, University of Kuopio, Finland, version 3.1.0.1) with time, frequency, and nonlinear domain analysis. A sampling rate of 1000 $\mathrm{Hz}$ was chosen, and recordings were transferred to a PC via v800 USB interface. Before the test exercise, each subject was instructed to lie on the exercise mat in a dimly controlled climate-controlled laboratory for 10 minutes following the adopted recommendations (Marek, 1996). The heart rate range was recorded five minutes before and five minutes after the test. The spectral response provided by the system was broken down into three bands: very low frequency $(0.003-0.04 \mathrm{~Hz})$, low frequency $(0.04-$ $0.15 \mathrm{~Hz})$, and high frequency $(0.15-0.4 \mathrm{~Hz})$. The nonlinear analysis techniques used in this study 
were the Poincare plot and detrended fluctuation analysis. The Poincaré diagrams were obtained by plotting the RR values of $n$ on the $x$-axis and the $R R$ values of $n+1$ on the $y$-axis. The SD1 axis indicates short-term variability, whereas the SD2 axis indicates long-term variability (Makivić, et al., 2013). Recordings were subsequently imported into Kubios HRV software (Biosignal Analysis and Medical Imaging Group, Department of Physics, University of Kuopio, Finland, version 3.1.0.1) (Tarvainen, Niskanen, Lipponen, Ranta-Aho, \& Karjalainen, 2014) for offline analyses. Specifically, we computed heart rate (beats per minute) as well as the mean of R-R intervals in milliseconds (Mean-RR), standard deviation of R-wave to R-wave intervals (SDNN) and root mean square of successive R-R intervals (RMSSD), the natural logarithmic transformation of the square root of the mean squared differences between successive R-R intervals in milliseconds (LnRMSSD), percentage of successive RR intervals that differ by more than $50 \mathrm{~ms}$ (PNN50), as three time-domain measures of heart rate variability with the absolute power of the low-frequency band (LF), absolute power of the high-frequency band (HF), and the ratio of LF- to HF-power (LF/HF).

\section{Statistical analysis}

Data are presented as means and standard deviations (SD). The differences based on magnitudes (Batterham \& Hopkins, 2006) were calculated to check the differences in the pre- and post-test moments. Sample size was calculated with G* power program (Ver.3.1.9.6) for a two-way ANOVA with repeated measures. Repeated two-factor analysis of variance (ANOVA) was used to compare HRV parameters in the participants of the two groups (3 times x 2 groups) for pre-test, test, and post-test. In order to compare the intra-group HRV parameters, a repeated one-way analysis of variance (ANOVA) was used during pre-test, test, and posttest. Binary comparisons were determined with Bonferroni test. The assumption of sphericity was tested using Mauchly's test and the GreenhouseGeiser correction factor to the degrees of freedom was used for all the positive tests. The effect size was calculated, which was considered small (0.01), medium $(0.06)$ or large $(>0.14)$. Eta-squared $\left(\mathrm{n}^{2}\right)$ is a measure of effect size for use in ANOVA. All calculations were made with SPSS version 22, statistical software (SPSS Inc., Chicago, IL, USA), and the level of significance was set at $\mathrm{p}<.05$.

\section{Results}

Physical characteristics of the participants and HRV values for 30 minutes sitting quietly are shown in Table 2.

A 3 (time) $x 2$ (group) ANOVA analyses for the $\mathrm{VO}_{2}$ max revealed a significant difference between
Table 2. Physical characteristics of the subjects $(n=53)$

\begin{tabular}{lc}
\hline Physical characteristics & Mean \pm SD \\
\hline Age (years) & $27.0 \pm 4.0$ \\
Height $(\mathrm{cm})$ & $177.9 \pm 5.7$ \\
Weight $(\mathrm{kg})$ & $77.8 \pm 8.7$ \\
BMI (kg.m $\left.{ }^{-2}\right)$ & $24.6 \pm 2.6$ \\
Body fat (\%) & $18.5 \pm 5.0$ \\
Mean-RR (bpm) & $682.9 \pm 212$ \\
SDNN (ms) & $58.5 \pm 38.6$ \\
\hline
\end{tabular}

the groups changes in the Mean-RR $\left(\mathrm{F}=3.626^{*}\right)$ and the ES was found to be large $\left(\eta^{2}=0.17\right)$. For the anaerobic test, between the groups changes of Mean-RR $\left(\mathrm{F}=3.768^{*}\right)$ and the ES was determined to be small $\left(\eta^{2}=0.01\right)$. Also, for the isokinetic strength test between the groups changes of Mean-RR $\left(\mathrm{F}=4.144^{*}\right)$ and the ES was determined to be small $\left(\eta^{2}=0.02\right)$.

For the $\mathrm{VO}_{2} \max$ test, there was a significant difference between the groups changes of SDNN $\left(\mathrm{F}=2.365^{*}\right)$, and the ES was determined to be small $\left(\eta^{2}=0.05\right)$. For the equilibrium test, between the groups changes of SDNN $(\mathrm{F}=5.379 *)$, and the ES was determined to be large $\left(\eta^{2}=0.13\right)$.

For the equilibrium test, there was a significant difference between the groups changes of RMSSD $\left(\mathrm{F}=4.599^{* * *}\right)$, and the ES was determined to be intermediate $\left(\eta^{2}=0.04\right)$.

For the $\mathrm{VO}_{2}$ max test, there was a significant difference between the groups changes of PNN50 $(\mathrm{F}=8.339 *)$ and the ES was determined to be small $\left(\mathrm{n}^{2}=0.05\right)$, and for the anaerobic test, there was a significant difference between the groups changes of PNN50 $(\mathrm{F}=5.312 *)$ and the ES was determined to be intermediate $\left(\mathrm{\eta}^{2}=0.10\right)$. Also, for the equilibrium test, there was a significant difference between the groups changes of PNN50 $\left(\mathrm{F}=4.487^{*}\right)$, and the ES was determined to be intermediate $\left(\eta^{2}=0.08\right)$.

For the $\mathrm{VO}_{2}$ max test, there was a significant difference between the groups changes of LF $\left(\mathrm{F}=4.520^{*}\right)$ and the ES was determined to be intermediate $\left(\mathrm{n}^{2}=0.08\right)$; for the equilibrium test there was a significant difference between the groups changes of $\operatorname{LF}\left(\mathrm{F}=8.407^{*}\right)$, and the ES was determined to be small $\left(\eta^{2}=0.04\right)$; and for the isokinetic strength test there was a significant difference between the groups changes of $\mathrm{LF}\left(\mathrm{F}=11.354^{*}\right)$, and the $\mathrm{ES}$ was determined to be small $\left(\eta^{2}=0.03\right)$.

For the $\mathrm{VO}_{2} \max$ test, there was a significant difference between the groups changes of HF $\left(\mathrm{F}=3.806^{*}\right)$ and the ES was determined to be small $\left(\mathrm{n}^{2}=0.02\right)$. For the anaerobic test, there was a significant difference between the groups changes of HF $\left(\mathrm{F}=7.395^{*}\right)$ and the ES was determined to be small $\left(\mathrm{n}^{2}=0.02\right)$, and for the equilibrium test, there was a significant difference between the groups changes 


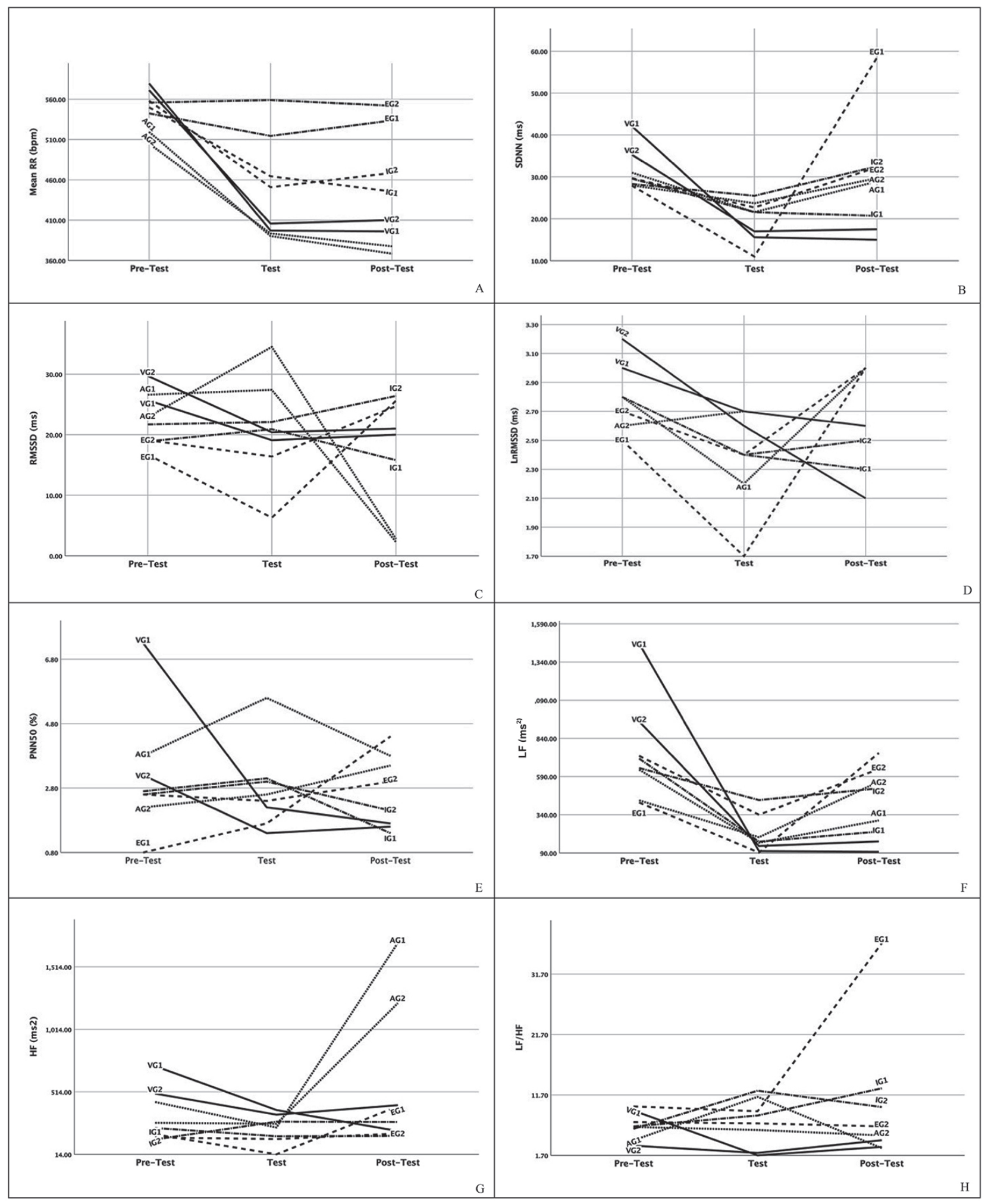

Figure 1. The change of HRV parameters of the groups in $\mathrm{VO}_{2} \max$, anaerobic, balance and isokinetic tests before, during and after the test. $\boldsymbol{V G 1}=G 1$ group's $V_{2}$ max mean values variation line. $\mathbf{V G 2}=G 1$ group's $V O_{2}$ max mean values variation line. $\boldsymbol{A G 1}=G 1$ group's anaerobic capacity mean values variation line. $\boldsymbol{A} \mathbf{G} \mathbf{2}=G 2$ group's anaerobic capacity mean values variation line. $\boldsymbol{E} \boldsymbol{G} \mathbf{1}=$ G1 group's equilibrium mean values variation line. $\mathbf{E} \boldsymbol{G} 2=G 2$ group's equilibrium mean values variation line. IG1=G1 group's isokinetic strength mean values variation line. IG2= G2 group's isokinetic strength mean values variation line.

of $\mathrm{HF}\left(\mathrm{F}=14.933^{*}\right)$ and the ES was determined to be intermediate $\left(\eta^{2}=0.08\right)$. Also, for the isokinetic strength test, there was a significant difference between the groups changes of $\mathrm{HF}\left(\mathrm{F}=9.745^{*}\right)$ and the ES was determined to be small $\left(\eta^{2}=0.02\right)$.
For the $\mathrm{VO}_{2}$ max test, there was a significant difference between the groups changes of LF/HF $\left(\mathrm{F}=8.603^{*}\right)$, and the $\mathrm{ES}$ was determined to be intermediate $\left(\mathrm{n}^{2}=0.06\right)$. For the equilibrium test, there was a significant difference between the groups 
changes of $\mathrm{LF} / \mathrm{HF}\left(\mathrm{F}=17.053^{*}\right)$ and the $\mathrm{ES}$ was determined to be small $\left(\eta^{2}=0.03\right)$. Also, for the isokinetic strength test, there was a significant difference between the groups changes of LF/HF $\left(\mathrm{F}=5.049^{*}\right)$ and the ES was determined to be small $\left(\mathrm{n}^{2}=0.01\right)$.

\section{Discussion and conclusions}

The study was conducted to investigate the availability of short-term and ultra-short-term HRV as a supportive tool to determine physical fitness. PubMed, Medline, and SPORTDiscus databases were examined before the study was planned. The novelty of this study is based on the lack of studies to investigate the relationship between short- and ultra-short-term HRV during different physical fitness tests.

Previously, HRV was used and measured during exercise (Lewis, Kingsley, Short, \& Simpson, 2007; Sandercock \& Brodie, 2006) alongside with before and after exercise. Millar, Rakobowchuk, McCartney, and MacDonald (2009) reported that WAnT may be useful in detecting changes in HRV frequency domain values more accurately in recovery after WAnT performed with one single and four multiples with 10 young male participants. Furthermore, HRV can be used as a performance diagnostic parameter in sports and as a control parameter of stress intensity (Hottenrott, Hoos, \& Esperer, 2006). Studies have shown that athletes with high HRV have superiority in competition (Nakamura, et al., 2015; Proietti et al., 2017). This can be explained in part by $\mathrm{VO}_{2}$ max found in athletes with better HRV values (Esco, Flatt, \& Nakamura, 2016). At the onset of exercise, heart rate increases mainly due to parasympathetic withdrawal (Goldsmith, Bloomfield, \& Rosenwinkel, 2000); this explains the dramatic decrease in HF power observed at the beginning of the exercise. Contradictory, sympathetic activity cannot be easily isolated by LF power (Marek, 1996). However, it is expected to increase with each subsequent training session because the increase in heart rate during exercise is a result of increased sympathetic stimulation (Goldsmith, et al., 2000). Differences from rest to exercise in HRV occur at the cell signal level at which activation of the $\beta 1$ receptor in cardiomyocytes increases HR and heart automation during exercise (Poehling, 2019).

In our study, G2 reached higher values in Mean$\mathrm{RR}$, one of the time-domain parameters, during and after the test in all the tests. This shows that recovery of autonomic nervous system (ANS) is faster for those with higher performance levels. Likewise, there were similar results in SDNN, but in G1 the SNNN increased dramatically in the equilibrium test after testing. We argue that this shows that stress factors of the participants also come to the fore during the test stages for the groups determined according to performance.
In the $\mathrm{VO}_{2}$ max test for the RMSSD parameter, which is primarily used for sportive performance, G2 started and finished with a higher value. In the anaerobic capacity test, RMSSD increased for G2 until the beginning of the test and decreased significantly in the two groups by the end of the test. In equilibrium and isokinetic strength tests, G2 completed again with a higher value. Tulppo, Makikallio, Seppänen, Laukkanen, and Huikuri, (1998) found that there was a relationship between the groups they formed according to physical performance, age, and cardiac vagal functions of the participants who had better physical fitness in bicycle test $\left(\mathrm{VO}_{2}\right.$ Peak). Since the RMSSD variable is frequently used in sports performance, it is also worth considering to look at the LnRMSSD value. While there were similar results for isokinetic capacity test with equilibrium in this variable, we obtained different results from RMSSD for the anaerobic capacity test with $\mathrm{VO}_{2}$ max. Logarithmic transformation led us to see different results for the present study. While the PNN50 value was higher in G1 than $\mathrm{G} 2$ before the $\mathrm{VO}_{2}$ max test, the two groups' values approached each other during the test and almost equalized after the test.

While the higher performance group had better values in aerobic and anaerobic capacity and isokinetic strength test results in time-domain variables, higher values were also found in the low performance group in the equilibrium test. Here, although G1 shows a worse equilibrium performance, it seems to be more effective in coping with stress.

One of the frequency domain parameters is the LF that showed a faster decrease in G1 than in $\mathrm{G} 2$ in tests requiring aerobic and anaerobic performance. G2 was higher after testing. But there was an opposite result in the equilibrium test. There were similar results in HF. However, after the anaerobic test, HF was much higher in G1. LF / HF value was much higher in G1 after the equilibrium test. To support this study, previous results showed that after exercise HRV values were higher, especially in subjects with better physical fitness (Melanson, 2000; Ostojic, Stojanovic, \& Calleja-Gonzalez, 2011; Seiler, et al., 2007; Somlev, Uzunova, \& Pavlova, 2012). However, Poehling (2019) did not find a significant relationship between Mean-RR and SDNN changes during submaximal and maximal tests. Our study observed that those with better performance in the equilibrium test did not have better HRV values. Aras and Coskun (2016) did not find any significant relationship between the changes in HRV values (SDNN, RMSSD, HF, LF / HF) before and after exercise. However, in another study, the intensity at the anaerobic threshold, 30 s after exercise, has been shown to primarily mediate parasympathetic reactivation (Imai, et al., 1994). We can say that the parasympathetic modulation of the group is better adapted with higher perfor- 
mance changes in the anaerobic test ultra-shortterm HRV values.

In previous studies on lower leg contraction with 23 male participants, significant changes in SDNN, RMSSD and HF values were found during dynamic strength exercises compared to static strength exercises, and explained that dynamic exercises lower HRV values more (Weippert, et al., 2013). Simões et al. (2016) concluded that leg maximum repetition (RM) and HRV are effective tools for estimating the lactate threshold in discontinuous resistance exercise, as a result of their study on subjects with cardiovascular disease. These results may impact the prescription of endurance resistance exercise in the cardiovascular disease population since cardiac vagal modulation is an essential indicator of cardiovascular protection and overactivity of sympathetic modulation is related to cardiovascular risk. No previous studies have been found similar to our research on isokinetic exercise.

As a result of our study, cardiac vagal functions reacted better in terms of short- and ultra-short-time HRV values in participants whose performance was higher than average, according to their test results. However, the equilibrium test has different results than the other tests. Except for the equilibrium test, we can accept our first hypothesis.

Our second hypothesis was that there would be a relationship between HRV values before, during and after the test. Previously, there were studies that monitor HRV changes before and after the test (Fortes, et al., 2017) or after the test (Mazzuco, et al., 2017). In our study, short-time and ultra-shorttime HRV changes were observed before, during and after the four different physical fitness tests. Poehling (2019) examined HRV (SDNN) values and submaximal and maximal HRV (SDNN) values from rest to submaximum and maximum exercise of 19-21 years old college runners. The researcher determined a significant difference in the study between rest and exercises. When comparing HRV between rest and exercise, the effect of the parasympathetic nervous system (PNS) at rest contributed to higher HRV, whereas both the submaximum and maximum sympathetic nervous system (SNS) effect during exercise corresponded to the reduced HRV. This is the first study to our knowledge to investigate the relationship between equilibrium ability and HRV values and further research is needed.

This study had some limitations. All of the participants were male. The effect of gender differences was not evaluated. Because the groups were divided according to their performance on the tests, one could expected performance differences depending on gender (Augustsson, et al., 2009; Cheuvront, Carter, Deruisseau, \& Moffatt, 2005). The lack of controlled breathing conditions may also be a limitation in HRV measurements. Especially in the $\mathrm{VO}_{2}$ max test and anaerobic capacity test because they are quite stressful tests. There was no control group in our research study; we grouped the participants according to their test results. We did not use all of the HRV parameters. Among these, we used time-domain (Mean-RR, SDNN-RMSSDLnRMSSD) and frequency domain (LF-HF-LF/HF) parameters, which are frequently tested in research. In ANS evaluation, besides heart rate, frequency domain variables such as VLF, total power (TP), and logarithmic transformation (Ln) and normalized units (nu) of almost all variables were used.

According to the present study results, as indicated in our first hypothesis, there is a significant difference between the performance of physical fitness tests and the changes of short- and ultrashort-term HRV parameters. HRV changes did not show parallelism with performance in the equilibrium test. Also, we accept our second hypothesis, especially since there was a significant difference between HRV changes before and after the tests. In the future, the effects of short-time and ultra-shorttime HRV parameters before, during and after exercise need to be tested with larger sample groups, different sexes and specific groups. Further studies are required to determine the relationship between isokinetic strength and equilibrium and HRV.

\section{References}

ACSM. (2013). ACSM's guidelines for exercise testing and prescription. Baltimore, MD: Lippincott Williams and Wilkins.

Aras, D., \& Coskun, B. (2016). The changes on the HRV after a Wingate anaerobic test in different simulated altitudes in healthy, physically-active adults. Acta Medica Mediterranea, 32, 1683-1688. doi: 10.19193/0393-6384_2016_5_150

Augustsson, S.R., Bersås, E., Thomas, E.M., Sahlberg, M., Augustsson, J., \& Svantesson, U. (2009). Gender differences and reliability of selected physical performance tests in young women and men. Advances in Physiotherapy, 11(2), 64-70. doi: 10.1080/14038190801999679

Baek, H.J., Cho, C.-H., Cho, J., \& Woo, J.M. (2015). Reliability of ultra-short-term analysis as a surrogate of standard 5-min analysis of heart rate variability. Telemedicine and e-Health, 21(5), 404-414. doi: 10.1089/tmj.2014.0104 
Bar-Or, O. (1987). The Wingate anaerobic test an update on methodology, reliability and validity. Sports Medicine, 4(6), 381-394. doi: 10.2165/00007256-198704060-00001

Batterham, A.M., \& Hopkins, W.G. (2006). Making meaningful inferences about magnitudes. International Journal of Sports Physiology and Performance, 1(1), 50-57. doi: 10.1123/ijspp.1.1.50

Berntson, G.G., Thomas Bigger Jr, J., Eckberg, D.L., Grossman, P., Kaufmann, P.G., Malik, M., Stone, P.H. (1997). Heart rate variability: Origins, methods, and interpretive caveats. Psychophysiology, 34(6), 623-648. doi: 10.1111/j.1469-8986.1997.tb02140.x

Buchheit, M., Chivot, A., Parouty, J., Mercier, D., Al Haddad, H., Laursen, P., \& Ahmaidi, S. (2010). Monitoring endurance running performance using cardiac parasympathetic function. European Journal of Applied Physiology, 108(6), 1153-1167. doi: 10.1007/s00421-009-1317-X

Chalencon, S., Busso, T., Lacour, J.R., Garet, M., Pichot, V., Connes, P., \& Barthélémy, J.C. (2012). A model for the training effects in swimming demonstrates a strong relationship between parasympathetic activity, performance and index of fatigue. PloS One, 7(12), e52636. doi: 10.1371/journal.pone.0052636

Cheuvront, S.N., Carter, R., Deruisseau, K.C., \& Moffatt, R.J. (2005). Running performance differences between men and women: An update. Sports Medicine, 35(12), 1017-1024. doi: 10.2165/00007256-200535120-00002

Driller, M.W., Argus, C.K., \& Shing, C.M. (2013). The reliability of a 30-s sprint test on the Wattbike cycle ergometer. International Journal of Sports Physiology and Performance, 8(4), 379-383. doi: 10.1123/ijspp.8.4.379

Esco, M., Flatt, A., \& Nakamura, F. (2016). Initial weekly HRV response is related to the prospective change in $\mathrm{VO}_{2} \mathrm{max}$ in female soccer players. International Journal of Sports Medicine, 37(6), 436-441. doi: 10.1055/s-0035-1569342

Fortes, L.S., da Costa, B.D., Paes, P.P., do Nascimento Júnior, J.R., Fiorese, L., \& Ferreira, M.E. (2017). Influence of competitive-anxiety on heart rate variability in swimmers. Journal of Sports Science and Medicine, 16(4), 498.

Franklin, B.A., Whaley, M.H., \& Howley, E.T. (Eds.) (2000). ACSM's guidelines for exercise testing and prescription. Baltimore, MD: Lippincott Williams and Wilkins.

Giles, D., \& Draper, N. (2018). Heart rate variability during exercise: A comparison of artefact correction methods. Journal of Strength and Conditioning Research, 32(3), 726-735. doi: 10.1519/JSC.0000000000001800

Giles, D., Draper, N., \& Neil, W. (2016). Validity of the Polar V800 heart rate monitor to measure RR intervals at rest. European Journal of Applied Physiology, 116(3), 563-571. doi: 10.1007/s00421-015-3303-9

Goldsmith, R.L., Bloomfield, D.M., \& Rosenwinkel, E.T. (2000). Exercise and autonomic function. Coronary Artery Disease, 11(2), 129-135. doi: 10.1097/00019501-200003000-00007

Hottenrott, K., Hoos, O., \& Esperer, H.D. (2006). Heart rate variability and physical exercise. Current status. Herz, 31(6), 544-552. doi: 10.1007/s00059-006-2855-1

Imai, K., Sato, H., Hori, M., Kusuoka, H., Ozaki, H., Yokoyama, H., \& Kamada, T. (1994). Vagally mediated heart rate recovery after exercise is accelerated in athletes but blunted in patients with chronic heart failure. Journal of the American College of Cardiology, 24(6), 1529-1535. doi: 10.1016/0735-1097(94)90150-3

Kiviniemi, A.M., Hautala, A.J., Kinnunen, H., \& Tulppo, M.P. (2007). Endurance training guided individually by daily heart rate variability measurements. European Journal of Applied Physiology, 101(6), 743-751. doi: 10.1007/ s00421-007-0552-2

Lewis, M.J., Kingsley, M., Short, A., \& Simpson, K. (2007). Rate of reduction of heart rate variability during exercise as an index of physical work capacity. Scandinavian Journal of Medicine and Science in Sports, 17(6), 696-702. doi: 10.1111/j.1600-0838.2006.00616.x

Makivić, B., Nikić Djordjević, M., \& Willis, M.S. (2013). Heart rate variability (HRV) as a tool for diagnostic and monitoring performance in sport and physical activities. Journal of Exercise Physiologyonline, 16(3), 103-131.

Manzi, V., Castagna, C., Padua, E., Lombardo, M., D’Ottavio, S., Massaro, M., Iellamo, F. (2009). Dose-response relationship of autonomic nervous system responses to individualized training impulse in marathon runners. American Journal of Physiology-Heart and Circulatory Physiology, 296(6), H1733-H1740. doi: 10.1152/ ajpheart.00054.2009

Marek, M., Bigger, J.T., Camm, A.J., Kleiger, R.E., Malliani, A., Moss, A.J., \& Schwartz, P.J. (1996). Heart rate variability: Standards of measurement, physiological interpretation, and clinical use. Circulation, 93(5), 10431065. doi: 10.1161/01.CIR.93.5.1043

Mazzuco, A., Medeiros, W.M., de Souza, A.S., Alencar, M.C.N., Neder, J.A., \& Borghi-Silva, A. (2017). Are heart rate dynamics in the transition from rest to submaximal exercise related to maximal cardiorespiratory responses in COPD? Brazilian Journal of Physical Therapy, 21(4), 251-258. doi: 10.1016/j.bjpt.2017.05.002

McNames, J., \& Aboy, M. (2006). Reliability and accuracy of heart rate variability metrics versus ECG segment duration. Medical and Biological Engineering and Computing, 44(9), 747-756. doi: 10.1007/s11517-006-0097-2

Melanson, E.L. (2000). Resting heart rate variability in men varying in habitual physical activity. Medicine and Science in Sports and Exercise, 32(11), 1894-1901. doi: 10.1097/00005768-200011000-00012

Millar, P.J., Rakobowchuk, M., McCartney, N., \& MacDonald, M.J. (2009). Heart rate variability and nonlinear analysis of heart rate dynamics following single and multiple Wingate bouts. Applied Physiology, Nutrition, and Metabolism, 34(5), 875-883. doi: 10.1139/H09-086

Mosley, E., \& Laborde, S. (2015). Performing with all my heart: Heart rate variability and its relationship with personality-trait-like-individual-differences (PTLIDs) in pressurized performance situations. In S. Walters 
(Ed.), Heart rate variability (HRV): Prognostic significance, risk factors and clinical applications (pp. 45-60). Hauppauge, NY: Nova Science Publishers.

Nakamura, F.Y., Flatt, A.A., Pereira, L.A., Ramirez-Campillo, R., Loturco, I., \& Esco, M.R. (2015). Ultra-shortterm heart rate variability is sensitive to training effects in team sports players. Journal of Sports Science and Medicine, 14(3), 602.

Nussinovitch, U., Elishkevitz, K.P., Katz, K., Nussinovitch, M., Segev, S., Volovitz, B., \& Nussinovitch, N. (2011). Reliability of ultra-short ECG indices for heart rate variability. Annals of Noninvasive Electrocardiology, 16(2), 117-122. doi: 10.1111/j.1542-474X.2011.00417.x.

Ostojic, S.M., Stojanovic, M.D., \& Calleja-Gonzalez, J. (2011). Ultra short-term heart rate recovery after maximal exercise: Relations to aerobic power in sportsmen. Chinese Journal of Physiology, 54(2), 105-110. doi: 10.4077/ CJP.2011.AMM018

Pichon, A.P., De Bisschop, C., Roulaud, M., Denjean, A., \& Papelier, Y. (2004). Spectral analysis of heart rate variability during exercise in trained subjects. Medicine and Science in Sports and Exercise, 36, 1702-1708. doi: 10.1249/01. MSS.0000142403.93205.35

Plews, D.J., Laursen, P.B., Kilding, A.E., \& Buchheit, M. (2012). Heart rate variability in elite triathletes, is variation in variability the key to effective training? A case comparison. European Journal of Applied Physiology, 112(11), 3729-3741. doi: 10.1007/s00421-012-2354-4

Poehling, C.P. (2019). The effects of submaximal and maximal exercise on heart rate variability. International Journal of Exercise Science, 12(2), 9-14.

Proietti, R., di Fronso, S., Pereira, L.A., Bortoli, L., Robazza, C., Nakamura, F.Y., \& Bertollo, M. (2017). Heart rate variability discriminates competitive levels in professional soccer players. Journal of Strength and Conditioning Research, 31(6), 1719-1725. doi: 10.1519/JSC.0000000000001795

Sandercock, G., \& Brodie, D. (2006). The use of heart rate variability measures to assess autonomic control during exercise. Scandinavian Journal of Medicine and Science in Sports, 16(5), 302-313. doi: 10.1111/j.16000838.2006.00556.x

Seiler, S., Haugen, O., \& Kuffel, E. (2007). Autonomic recovery after exercise in trained athletes: Intensity and duration effects. Medicine and Science in Sports and Exercise, 39(8), 1366-1373. doi: 10.1249/mss.0b013e318060f17d

Simões, R.P., Mendes, R.G., Castello-Simões, V., Catai, A.M., Arena, R., \& Borghi-Silva, A. (2016). Use of heart rate variability to estimate lactate threshold in coronary artery disease patients during resistance exercise. Journal of Sports Science and Medicine, 15(4), 649.

Somlev, P., Uzunova, G., \& Pavlova, E. (2012). Heart rate variability at rest in elite and former soccer players. Scripta Scientifica Medica, 44, 89-91. doi: 10.1055/s-0035-1550047

Taralov, Z.Z., Terziyski, K.V., \& Kostianev, S.S. (2015). Heart rate variability as a method for assessment of the autonomic nervous system and the adaptations to different physiological and pathological conditions. Folia Medica, 57(3-4), 173-180. doi: 10.1515/folmed-2015-0036

Tarvainen, M.P., Niskanen, J.P., Lipponen, J.A., Ranta-Aho, P.O., \& Karjalainen, P.A. (2014). Kubios HRV-heart rate variability analysis software. Computer Methods and Programs in Biomedicine, 113(1), 210-220. doi: 10.1016/j. cmpb.2013.07.024

Tomes, C., Schram, B., \& Orr, R. (2020). Relationships between heart rate variability, occupational performance, and fitness for tactical personnel: A systematic review. Frontiers in Public Health, 8, 583336). doi: 10.3389/ fpubh.2020.583336

Tulppo, M.P., Makikallio, T.H., Seppänen, T., Laukkanen, R.T., \& Huikuri, H.V. (1998). Vagal modulation of heart rate during exercise: Effects of age and physical fitness. American Journal of Physiology-Heart and Circulatory Physiology, 274(2), H424-H429. doi: 10.1152/ajpheart.1998.274.2.H424

Weippert, M., Behrens, K., Rieger, A., Stoll, R., \& Kreuzfeld, S. (2013). Heart rate variability and blood pressure during dynamic and static exercise at similar heart rate levels. PloS One, 8(12), e83690. doi: 10.1371/journal. pone.0083690

Zupan, M.F., Arata, A.W., Dawson, L.H., Wile, A.L., Payn, T.L., \& ve Hannon, M.E. (2009). Wingate anaerobic test peak power and anaerobic capacity classifications for men and women intercollegiate athletes. Journal of Strength and Conditioning Research, 23(9), 2598-2604.

Submitted: November 20, 2019

Accepted: April 4, 2021

Published Online First: May 27, 2021

Correspondence to:

Tuncay Alparslan, Ph.D.

Atatürk St. 1726030 Odunpazarı/ESKİŞEHİR.

Phone: +90 (0222) 324-0540

E-mail: talparslan@hvkk.tsk.tr 